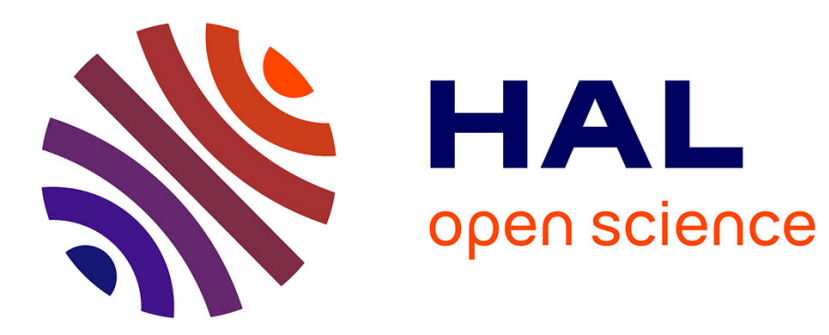

\title{
Put option prices as joint distribution functions in strike and maturity: the Black-Scholes case
}

\author{
D. Madan, Bernard Roynette, Marc Yor
}

\section{To cite this version:}

D. Madan, Bernard Roynette, Marc Yor. Put option prices as joint distribution functions in strike and maturity: the Black-Scholes case. International Journal of Theoretical and Applied Finance, 2009, 12 (8), pp.1075-1090. 10.1142/S0219024909005580 . hal-00324636

\section{HAL Id: hal-00324636 \\ https://hal.science/hal-00324636}

Submitted on 25 Sep 2008

HAL is a multi-disciplinary open access archive for the deposit and dissemination of scientific research documents, whether they are published or not. The documents may come from teaching and research institutions in France or abroad, or from public or private research centers.
L'archive ouverte pluridisciplinaire HAL, est destinée au dépôt et à la diffusion de documents scientifiques de niveau recherche, publiés ou non, émanant des établissements d'enseignement et de recherche français ou étrangers, des laboratoires publics ou privés. 


\title{
Put option prices as joint distribution functions
}

\section{in strike and maturity : the Black-Scholes case}

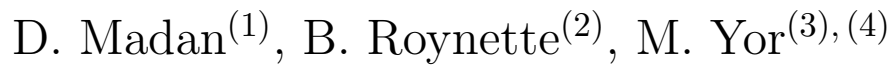

$05 / 09 / 2008$

(1) Robert H. Smith School of Business, Van Munching Hall, University of Maryland, College Park, MD. 20742 USA

email : dbm@rhsmith.umd.edu

(2) Institut Elie Cartan, Université Henri Poincaré, B.P. 239, 54506 Vandoeuvre les Nancy Cedex email : bernard.roynette@iecn.u-nancy.fr

(3) Laboratoire de Probabilités et Modèles Aléatoires, Université Paris VI et VII, 4 place Jussieu - Case 188

F - 75252 Paris Cedex 05

email : deaproba@proba.jussieu.fr

(4) Institut Universitaire de France

Abstract : For a large class of $\mathbb{R}_{+}$valued, continuous local martingales $\left(M_{t} t \geq 0\right)$, with $M_{0}=1$ and $M_{\infty}=0$, the put quantity : $\Pi_{M}(K, t)=E\left(\left(K-M_{t}\right)^{+}\right)$turns out to be the distribution function in both variables $K$ and $t$, for $K \leq 1$ and $t \geq 0$, of a probability $\gamma_{M}$ on $[0,1] \times[0, \infty[$. In this paper, the first in a series of three, we discuss in detail the case where $M_{t}=\mathcal{E}_{t}:=\exp \left(B_{t}-\frac{t}{2}\right)$, for $\left(B_{t}, t \geq 0\right)$ a standard Brownian motion.

Keywords : First and last passage times, pseudo-inverse, local time-space calculus, BlackScholes set up.

\section{Introduction}

1.1 Throughout this paper, we consider a generic continuous local martingale $\left(M_{t}, t \geq 0\right)$, taking values in $\mathbb{R}_{+}$, and such that :

$$
M_{0}=1, \lim _{t \rightarrow \infty} M_{t}=0 \quad \text { a.s. }
$$


To such an $\left(M_{t}, t \geq 0\right)$, we associate the function ${ }^{1} \Pi_{M}:[0,1] \times \mathbb{R}_{+} \rightarrow \mathbb{R}_{+}$defined by :

$$
\Pi_{M}(K, t):=E\left(\left(K-M_{t}\right)^{+}\right), \quad 0 \leq K \leq 1, t \geq 0
$$

Note that this function is separately increasing in $K$ and $t$ (since, $(K-x)^{+}$being convex, $\left(\left(K-M_{t}\right)^{+}, t \geq 0\right)$ is a submartingale). Furthermore, we have :

(i) $\quad \Pi_{M}(K, 0)=0 \quad(0 \leq K \leq 1) \quad$ and

$$
\Pi_{M}(K, \infty):=\lim _{t \rightarrow \infty} \Pi_{M}(K, t)=K
$$

(from the dominated convergence Theorem)

(ii) $\quad \Pi_{M}(K, t)=K P\left(\mathcal{G}_{K} \leq t\right)$

with $\quad \mathcal{G}_{K}:=\sup \left\{t \geq 0 ; M_{t}=K\right\}$

(see [1], [5], [6], [7], [8]). In particular :

$$
\Pi_{M}(1, t)=P\left(\mathcal{G}_{1} \leq t\right)
$$

Thus, $\left(\Pi_{M}(K, \infty), K \leq 1\right)$, resp. $\left(\Pi_{M}(1, t), t \geq 0\right)$ is a distribution function on $[0,1]$, resp. on $[0, \infty[;$ more precisely, these functions are, respectively, the distribution function of $U$, a standard uniform variable on $[0,1]$, and of $\mathcal{G}_{1}$.

To illustrate (1.6), let us recall that in the case $\left(M_{t}=\mathcal{E}_{t}:=\exp \left(B_{t}-\frac{t}{2}\right), t \geq 0\right)$, with $\left(B_{t} ; t \geq 0\right)$ a standard Brownian motion it was shown in [5] that : $\mathcal{G}_{1} \stackrel{\text { (law) }}{=} 4 B_{1}^{2}$; hence, (1.6) reads, in this case :

$$
\Pi_{\mathcal{E}}(1, t)=P\left(4 B_{1}^{2} \leq t\right)
$$

This formula may also be checked directly from the Black-Scholes formula.

1.2 It is thus a natural question to ask whether the function of $K$ and $t$ :

$\left(\Pi_{M}(K, t) ; K \leq 1, t \geq 0\right)$ is the distribution function of a probability on $[0,1] \times[0, \infty[$ which, assuming it exists, we shall denote by $\gamma\left(=\gamma_{M}\right)$. If so, we have :

$$
E\left(\left(K-M_{t}\right)^{+}\right)=\gamma([0, K] \times[0, t]), \quad K \leq 1, t \geq 0
$$

1.3 Here is our strategy to attack this question. Note that by Fubini :

$$
E\left(\left(K-M_{t}\right)^{+}\right)=\int_{0}^{K} P\left(M_{t} \leq x\right) d x
$$

\footnotetext{
${ }^{1}$ It is the "put" associated to the local martingale $\left(M_{t}\right)$, with strike $K$, and maturity $t$.
} 
Assume that there exists, for every $x<1$, a r.v. $Y_{x} \geq 0$ such that:

$$
P\left(M_{t} \leq x\right)=P\left(Y_{x} \leq t\right) \quad(x<1, t \geq 0)
$$

We shall call this collection $\left(Y_{x}, x \leq 1\right)$ of r.v.'s, provided it exists, a pseudo-inverse of $\left(M_{t}, t \geq 0\right)$.

To justify this terminology, think of $\left(M_{t}, t \geq 0\right)$ as a process with a tendency to decrease, since it is a supermartingale which converges to 0 as $t \rightarrow \infty$. Now, replace $\left(M_{t}, t \geq 0\right)$ by a decreasing process $\left(D_{t}, t \geq 0\right)$; then, if $\left(\theta_{d}, d \geq 0\right)$ is the inverse of $D$, we have : $P\left(D_{t} \leq x\right)=P\left(\theta_{x} \leq t\right)$.

Let us go back to (1.9), and assume that $\left(M_{t}, t \geq 0\right)$ admits a pseudo-inverse $\left(Y_{x}, x \leq 1\right)$. Then, plugging (1.10) in (1.9), we find that $\gamma$ exists, and it is the probability :

$$
\gamma(d x, d t)=d x P\left(Y_{x} \in d t\right) \quad \text { on }[0,1] \times[0, \infty[
$$

Note that, a priori, we do not know the existence of $\left(Y_{x}, x \leq 1\right)$ as a process ; if such a process exists, then $\gamma$ is the law of :

$$
\left(U, Y_{U}\right)
$$

where $U$ is uniform on $[0,1]$, independent of $\left(Y_{x}, x<1\right)$.

1.4 In practice, most of the time, the function :

$$
(K, t) \rightarrow \Pi_{M}(K, t)
$$

is regular ; if so, we find that $\left(M_{t}, t \geq 0\right)$ admits a pseudo-inverse if and only if :

$$
\frac{\partial^{2}}{\partial K \partial t}\left(\Pi_{M}(K, t)\right) \geq 0
$$

and then :

$$
\gamma(d K, d t)=d K P\left(Y_{K} \in d t\right)=\left(\frac{\partial^{2}}{\partial K \partial t}\left(\Pi_{M}(K, t)\right)\right) d K d t
$$

1.5 In Section 2, we shall develop this program in the case $M_{t}=\mathcal{E}_{t}:=$ $\exp \left(B_{t}-\frac{t}{2}\right), t \geq 0$ where $\left(B_{t}, t \geq 0\right)$ is a standard Brownian motion started at 0 . In particular, we prove the existence of a pseudo-inverse for $\left(\mathcal{E}_{t}, t \geq 0\right)$.

1.6 In Part II, (in preparation) we aim to show the existence of a pseudo-inverse for a large number of Markovian martingales $\left(M_{t}, t \geq 0\right)$. In Part III, further classes of martingales shall be shown to admit pseudo-inverses. 


\section{The Black-Scholes paradigm}

2.1 In this Section, $\left(B_{t}, t \geq 0\right)$ denotes a standard Brownian motion started at 0 and $\left(\mathcal{E}_{t}, t \geq 0\right)$ is the exponential martingale defined by :

$$
\mathcal{E}_{t}:=\exp \left(B_{t}-\frac{t}{2}\right), \quad t \geq 0
$$

Note that $\mathcal{E}_{0}=1$ and $\mathcal{E}_{t} \underset{t \rightarrow \infty}{\longrightarrow} 0$ a.s. We define, for $0 \leq K \leq 1$ and $t \geq 0$ :

$$
\Pi_{\mathcal{E}}(K, t):=E\left(\left(K-\mathcal{E}_{t}\right)^{+}\right)
$$

Theorem 2.1 There exists a probability, which we shall denote by $\gamma$, on $[0,1] \times[0, \infty[$ such that :

$$
\Pi_{\mathcal{E}}(K, t)=\gamma([0, K] \times[0, t])
$$

(see point ii) of Proposition 2.7 for a description of the density of $\gamma$ ).

In order to prove Theorem 2.1 and to describe $\gamma$, we start with :

Lemma 2.2 Denote by $\mathcal{N}$ the distribution function of the standard Gaussian variable:

$$
\mathcal{N}(x):=\frac{1}{\sqrt{2 \pi}} \int_{-\infty}^{x} e^{-\frac{y^{2}}{2}} d y \quad(x \in \mathbb{R})
$$

Then :

i) To any $a, b>0$, one can associate a r.v. $Y_{a, b}$, taking values in $[0, \infty[$, such that :

$$
P\left(Y_{a, b} \leq t\right)=\mathcal{N}\left(a \sqrt{t}-\frac{b}{\sqrt{t}}\right), \quad t \geq 0
$$

ii) The density $f_{Y_{a, b}}$ of $Y_{a, b}$ is given by:

$$
f_{Y_{a, b}}(t)=\frac{1}{\sqrt{2 \pi}} e^{a b} \cdot\left(\frac{a}{2 \sqrt{t}}+\frac{b}{2 t^{\frac{3}{2}}}\right) \exp \left(-\frac{1}{2}\left(a^{2} t+\frac{b^{2}}{t}\right)\right)
$$

iii) Let us define :

$$
\begin{aligned}
& T_{b}^{(a)}:=\inf \left\{t \geq 0 ; B_{t}+a t=b\right\} \\
& G_{b}^{(a)}:=\sup \left\{t \geq 0 ; B_{t}+a t=b\right\}
\end{aligned}
$$

Then :

$$
P\left(Y_{a, b} \in d t\right)=\frac{1}{2}\left\{P\left(T_{b}^{(a)} \in d t\right)+P\left(G_{b}^{(a)} \in d t\right)\right\}
$$


We note that this formula (2.9) allows to define the law of a process $\left(Y_{a, b} ; b \geq 0\right)$ obtained as a fair coin toss of $\left(T_{b}^{(a)} ; b \geq 0\right)$ on one hand and $\left(G_{b}^{(a)} ; \overline{b \geq 0)}\right.$ on the other hand.

\section{Remark 2.3}

i) It may be worth mentioning that this point $i$ ) of Lemma 2.2 admits a wide extension, since to any distribution function $F$ on $\mathbb{R}$, and any $a, b>0$, we can associate a new distribution function $F_{a, b}$ on $\mathbb{R}_{+}$via :

$$
F_{a, b}(t)=F\left(a \sqrt{t}-\frac{b}{\sqrt{t}}\right) \quad(t \geq 0)
$$

However, the particular case $F=\mathcal{N}$ fits extremely well with our discussion.

ii) We note that $\frac{1}{Y_{a, b}} \stackrel{\text { (law) }}{=} Y_{b, a}$, which may be deduced from either (2.5), (2.6) or (2.9).

\section{Proof of Lemma 2.2}

a. Points i) and ii) of Lemma 2.2 are immediate since :

$$
\begin{aligned}
\text { - } \quad \lim _{t \downarrow 0} \mathcal{N}\left(a \sqrt{t}-\frac{b}{\sqrt{t}}\right) & =0 \\
\text { - } \quad \lim _{t \uparrow \infty} \mathcal{N}\left(a \sqrt{t}-\frac{b}{\sqrt{t}}\right) & =1 \\
\text { - } \quad \frac{\partial}{\partial t} \mathcal{N}\left(a \sqrt{t}-\frac{b}{\sqrt{t}}\right) & =\frac{1}{\sqrt{2 \pi}}\left(\frac{a}{2 \sqrt{t}}+\frac{b}{2 t^{\frac{3}{2}}}\right) \exp -\frac{1}{2}\left(a \sqrt{t}-\frac{b}{\sqrt{t}}\right)^{2} \geq 0 \\
& =f_{Y_{a, b}}(t)
\end{aligned}
$$

b. We now prove point iii) of Lemma 2.2 :

We shall use the well-known formulae (see e.g. [6])

$$
\begin{aligned}
& P\left(T_{b}^{(a)} \in d t\right)=\frac{b d t}{\sqrt{2 \pi t^{3}}} \exp -\frac{1}{2 t}(b-a t)^{2} \quad(a, b>0, t \geq 0) \\
& P\left(G_{b}^{(a)} \in d t\right)=\frac{a d t}{\sqrt{2 \pi t}} \exp -\frac{1}{2 t}(b-a t)^{2} \quad(a, b>0, t \geq 0)
\end{aligned}
$$

(2.9) is now an immediate consequence of (2.10), (2.11) and (2.6).

c. Another proof of point iii) of Lemma 2.2 :

Let us denote, for $\nu>0,\left(\mathcal{E}_{t}^{(2 \nu)}:=\exp \left(2 \nu B_{t}-2 \nu^{2} t\right), t \geq 0\right)$. It is proven in [6] Theorem 1, that, for $A \geq 1$ :

$$
P\left(T_{\log A}^{(\nu)} \leq t\right)=E\left(\mathcal{E}_{t}^{(2 \nu)} 1_{\mathcal{E}_{t}^{(2 \nu)}>A^{2 \nu}}\right)+A^{2 \nu} P\left(\mathcal{E}_{t}^{(2 \nu)}>A^{2 \nu}\right)
$$

and for $A \geq 0$ :

$$
P\left(G_{\log A}^{(\nu)} \leq t\right)=E\left(\mathcal{E}_{t}^{(2 \nu)} 1_{\mathcal{E}_{t}^{(2 \nu)}>A^{2 \nu}}\right)-A^{2 \nu} P\left(\mathcal{E}_{t}^{(2 \nu)}>A^{2 \nu}\right)
$$


Thus, by addition :

$$
\begin{aligned}
\frac{1}{2}\left\{P\left(T_{\log A}^{(\nu)} \leq t\right)\right. & \left.+P\left(G_{\log A}^{(\nu)} \leq t\right)\right\}=E\left(\mathcal{E}_{t}^{(2 \nu)} 1_{\mathcal{E}_{t}^{(2 \nu)}>A^{2 \nu}}\right) \\
& =P\left(e^{2 \nu B_{t}+2 \nu^{2} t}>A^{2 \nu}\right) \quad(\text { from Cameron-Martin formula) } \\
& =P\left(B_{t}>\log A-\nu t\right)=P\left(B_{1}>\frac{\log A-\nu t}{\sqrt{t}}\right) \\
& =1-\mathcal{N}\left(\frac{\log A}{\sqrt{t}}-\nu \sqrt{t}\right)=\mathcal{N}\left(\nu \sqrt{t}-\frac{\log A}{\sqrt{t}}\right)
\end{aligned}
$$

(2.6) is now an immediate consequence of (2.12), with $b=\log A$ and $\nu=a$, by derivation with respect to $t$.

Proof of Theorem 2.1 From (1.8), we denote, for $K \leq 1$ and $t \geq 0$ :

$$
\begin{aligned}
\Pi_{\mathcal{E}}(K, t) & :=E\left(\left(K-\mathcal{E}_{t}\right)^{+}\right)=\int_{0}^{K} P\left(\mathcal{E}_{t} \leq x\right) d x \\
& =\int_{0}^{K} \mathcal{N}\left(\frac{\log x}{\sqrt{t}}+\frac{\sqrt{t}}{2}\right) d x \\
\text { since } P\left(\mathcal{E}_{t} \leq x\right) & \left.=P\left(e^{B_{t}-\frac{t}{2}} \leq x\right)=P\left(B_{1}<\frac{\log x}{\sqrt{t}}+\frac{\sqrt{t}}{2}\right)\right) \\
& =\int_{0}^{K} P\left(Y_{\frac{1}{2}}, \log \frac{1}{x} \leq t\right) d x \quad(\text { from Lemma 2.2) }
\end{aligned}
$$

Hence :

$$
\frac{\partial^{2}}{\partial K \partial t} \Pi_{\mathcal{E}}(K, t)=\frac{\partial}{\partial t} P\left(Y_{\frac{1}{2}, \log \frac{1}{K}} \leq t\right)=f_{Y_{\frac{1}{2}, \log \frac{1}{K}}}(t) \geq 0
$$

This ends the proof of Theorem 1.

\subsection{Descriptions of the probability $\gamma$}

2.2.1 First description of $\gamma$ : conditioning with respect to $U$

Proposition 2.4 The probability $\gamma$ on $[0,1] \times[0, \infty[$ is the law of the pair :

$$
\left(U, Y_{\frac{1}{2}}, \log \frac{1}{U}\right)
$$

where $U$ is uniform on $[0,1]$, independent of the process $\left(Y_{\frac{1}{2}, b}, b>0\right)$.

We now describe in words this probability viewed from (2.14) : it is the law of a two components r.v. ; the first component is the choice of a level out ot the moneyness for a put, or the choice of a strike $K<1$, uniformly on [0,1]. Given this level, we construct the second variable on the outcome of a fair coin toss as either the first passage time of 
the stock price under the share measure to the level $\left(\frac{1}{K}\right)$, or the last passage time of the stock price under the share measure to level $\left(\frac{1}{K}\right)$.

\section{Proof of Proposition 2.4}

We have, for $K \leq 1$ and $t \geq 0$ :

$$
\begin{aligned}
& P\left(U \leq K, Y_{\frac{1}{2}}, \log \frac{1}{U} \leq t\right)=\int_{0}^{K} d u P\left(Y_{\frac{1}{2}}, \log \frac{1}{u} \leq t\right) \quad \text { (as explained just above) } \\
& =\int_{0}^{K} \mathcal{N}\left(\frac{\sqrt{t}}{2}+\frac{\log u}{\sqrt{t}}\right) d u \\
& =\int_{0}^{K} P\left(\mathcal{E}_{t} \leq u\right) \quad\left(\text { since } \mathcal{E}_{t} \stackrel{\text { (law) }}{=} \exp \left(\sqrt{t} B_{1}-\frac{t}{2}\right) \text {, for fixed } t\right) \\
& =E\left[\left(K-\mathcal{E}_{t}\right)^{+}\right] \quad(\text { from }(1.9)) \\
& =\gamma([0 K] \times[0, t]) \quad(\text { from }(2.3))
\end{aligned}
$$

The density of $\gamma$ with respect to the Lebesgue measure on $[0,1] \times \mathbb{R}_{+}$given by (2.23) may also be obtained from the preceding relations.

2.2.2 Second description of $\gamma$ : conditioning with respect to $\mathcal{G}_{1}$

Proposition 2.5 The probability $\gamma$ on $[0,1] \times[0, \infty[$ is the law of the pair :

$$
\left(\exp (-2 \mathfrak{e}) \vee \exp \left(-\sqrt{8 \mathfrak{e}^{\prime} B_{1}^{2}}\right), \quad 4 B_{1}^{2}\right)
$$

where $B_{1}, \mathfrak{e}, \mathfrak{e}^{\prime}$ are independent, with $\mathfrak{e}$ and $\mathfrak{e}^{\prime}$ two standard exponential variables.

Remark 2.6 Upon comparing Propositions 2.4 and 2.5, it is quite natural to look for some understanding of the implied identities in laws between the first, resp. second, components of (2.15) and (2.14) ; precisely, we wish to check directly that:

$$
\begin{gathered}
(\exp (-2 \mathfrak{e})) \vee\left(\exp \left(-\sqrt{8 \mathfrak{e}^{\prime} B_{1}^{2}}\right)\right) \stackrel{(\text { law })}{=} U \\
Y_{\frac{1}{2}}, \log \frac{1}{U} \stackrel{\text { (law })}{=} 4 B_{1}^{2} \quad\left(\stackrel{(\text { law })}{=} \mathcal{G}_{0}^{\left(-\frac{1}{2}\right)}\right)
\end{gathered}
$$

a. We now prove $(2.16)$

- First, we have :

$$
\sqrt{2 \mathfrak{e}^{\prime} B_{1}^{2}} \stackrel{(\text { law })}{=} \mathfrak{e}
$$

Indeed :

$$
P\left(\sqrt{2 \mathfrak{e}^{\prime} B_{1}^{2}}>x\right)=P\left(\mathfrak{e}^{\prime}>\frac{x^{2}}{2 B_{1}^{2}}\right)=E\left(\exp \left(-\frac{x^{2}}{2 B_{1}^{2}}\right) \stackrel{(a)}{=} E\left(\exp \left(-\frac{x^{2}}{2} T_{1}\right)\right) \stackrel{(b)}{=} \exp (-x)\right.
$$


since $T_{1}$, the first hitting time of 1 by $\left(B_{t}, t \geq 0\right)$ is distributed as $\frac{1}{B_{1}^{2}}$, hence $(a)$, and the Laplace transform of $T_{1}$ is well known to be given by $(b)$.

- Since $\exp (-2 \mathfrak{e}) \stackrel{\text { (law) }}{=} U^{2}$, we have, from $(2.18)$ :

$$
(\exp (-2 \mathfrak{e})) \vee\left(\exp \left(-\sqrt{8 \mathfrak{e}^{\prime} B_{1}^{2}}\right)\right) \stackrel{(\text { law })}{=} U^{2} \vee\left(U^{\prime}\right)^{2}
$$

with $U$ and $U^{\prime}$ uniform on $[0,1]$ and independent. But, for $y \in[0,1]$ :

$$
P\left(U^{2} \vee\left(U^{\prime}\right)^{2} \leq y\right)=\left[P\left(U^{2} \leq y\right)\right]^{2}=(\sqrt{y})^{2}=y
$$

We have proven (2.16).

b. We now prove (2.17)

We have for every $t \geq 0$ :

$$
\begin{aligned}
& \left.P\left(Y_{\frac{1}{2}, \log \frac{1}{U}} \leq t\right)=\int_{0}^{1} P\left(Y_{\frac{1}{2}, \log \frac{1}{u}} \leq t\right) d u \quad \text { (after conditioning by } U=u\right) \\
& =\int_{0}^{1} \mathcal{N}\left(\frac{\sqrt{t}}{2}+\frac{\log u}{\sqrt{t}}\right) d u \quad(\text { from Lemma } 2.2) \\
& =E\left(\left(1-\mathcal{E}_{t}\right)^{+}\right) \quad(\text { from }(2.13)) \\
& =P\left(4 B_{1}^{2} \leq t\right) \quad(\text { from the classical Black-Scholes formula, see }(1.7))
\end{aligned}
$$

\section{We now prove Proposition 2.5}

Conditioning on $B_{1}^{2}$ and using the explicit formula for the density of $B_{1}^{2}$ :

$$
f_{B_{1}^{2}}(z)=\frac{1}{\sqrt{2 \pi z}} e^{-\frac{z}{2}} 1_{z>0}
$$

we have, for $K \leq 1$ and $t \geq 0$ :

$$
\begin{aligned}
& \left.P(\exp (-2 \mathfrak{e})) \vee\left(\exp -\sqrt{8 \mathfrak{e}^{\prime} B_{1}^{2}}\right) \leq K, 4 B_{1}^{2} \leq t\right) \\
& \quad=\int_{0}^{\frac{t}{4}} \frac{d z}{\sqrt{2 \pi z}} e^{-\frac{z}{2}} P\left((\exp (-2 \mathfrak{e})) \vee\left(\exp -z \sqrt{8 \mathfrak{e}^{\prime}}\right) \leq K\right) \\
& \quad=\int_{0}^{\frac{t}{4}} \frac{d z}{\sqrt{2 \pi z}} e^{-\frac{z}{2}} P((\exp (-2 \mathfrak{e})) \leq K) P\left(\mathfrak{e}^{\prime}>\frac{\log ^{2}\left(\frac{1}{K}\right)}{8 z^{2}}\right) \\
& \quad=\sqrt{K} \int_{0}^{\frac{t}{4}} \frac{d z}{\sqrt{2 \pi z}} e^{-\frac{z}{2}} \exp \left(-\frac{\left(\log \frac{1}{K}\right)^{2}}{8 z^{2}}\right) \quad(\text { since } \exp (-\mathfrak{e}) \stackrel{(\text { law })}{=} U) \\
& =\sqrt{K} E\left(1_{B_{1}^{2} \leq \frac{t}{4}} \cdot \exp -\frac{\log ^{2}\left(\frac{1}{K}\right)}{8 B_{1}^{2}}\right) \\
& \quad=E\left[\left(K-\mathcal{E}_{t}\right)^{+}\right]
\end{aligned}
$$


where the last equality follows from Theorem 2 of [6] which asserts that for $K \leq 1$ :

$$
E\left[\left(K-\mathcal{E}_{t}\right)^{+}\right]=\sqrt{K} E\left\{1_{4 B_{1}^{2} \leq t} \exp \left(-\frac{\left(\log \left(\frac{1}{K}\right)\right)^{2}}{8 B_{1}^{2}}\right)\right\}
$$

\section{Another proof of Proposition 2.5}

We have, from (2.11) with $a=1 / 2$ and $K<1$ :

$$
P\left(\mathcal{G}_{K} \in d s\right)=\frac{\frac{1}{2}}{\sqrt{2 \pi s}} \exp -\frac{1}{2 s}\left(\log K+\frac{s}{2}\right)^{2} d s
$$

Hence :

$$
\begin{aligned}
K P\left(\mathcal{G}_{K} \in d s\right) & =\sqrt{K}\left(\exp \left(-\frac{\left(\log \frac{1}{K}\right)^{2}}{2 s}\right)\right) P\left(\mathcal{G}_{1} \in d s\right) \\
& =\exp \left(\frac{1}{2} \log K\right) \exp \left(-\frac{\left(\log \left(\frac{1}{K}\right)\right)^{2}}{2 s}\right) P\left(\mathcal{G}_{1} \in d s\right) \\
& =P\left(\mathfrak{e}>-\frac{1}{2} \log K\right) P\left(\mathfrak{e}^{\prime}>\frac{\left(\log \frac{1}{K}\right)^{2}}{2 s}\right) P\left(\mathcal{G}_{1} \in d s\right) \\
& =P\left(\exp (-2 \mathfrak{e}) \vee \exp \left(-\sqrt{2 s \mathfrak{e}^{\prime}}\right)<K\right) P\left(\mathcal{G}_{1} \in d s\right) \\
& =P\left(\exp (-2 \mathfrak{e}) \vee \exp \left(-\sqrt{8 B_{1}^{2} \mathfrak{e}^{\prime}}\right)<K\right) P\left(4 B_{1}^{2} \in d s\right)
\end{aligned}
$$

since $\mathcal{G}_{1} \stackrel{\text { law) }}{=} 4 B_{1}^{2}($ see [5]).

Hence, since, from $(1.4), E\left(\left(K-\mathcal{E}_{s}\right)^{+}\right)=K P\left(\mathcal{G}_{K} \leq s\right)$, one has :

$$
\begin{aligned}
\gamma(d K, d s) & =\frac{\partial^{2}}{\partial K \partial s}\left(K P\left(\mathcal{G}_{K} \leq s\right)\right) d K d s \\
& =P\left(\exp (-2 \mathfrak{e}) \vee \exp \left(-\sqrt{8 B_{1}^{2} \mathfrak{e}^{\prime}}\right) \in d K\right) P\left(4 B_{1}^{2} \in d s\right)
\end{aligned}
$$

which is Proposition 2.5

2.2.3 Third description of $\gamma$ : its relation with local time-space calculus Let us define $\left(L_{s}^{K} ; K \geq 0, s \geq 0\right)$ the jointly continuous family of local times of the martingale $\left(\mathcal{E}_{s}, s \geq 0\right)$. This family is characterized by the occupation density formula :

$$
\int_{0}^{t} f\left(\mathcal{E}_{s}\right) d<\mathcal{E}>_{s}=\int_{0}^{\infty} f(K) L_{t}^{K} d K
$$


for every Borel and positive function $f$. Here $\left(\langle\mathcal{E}\rangle_{s}, s \geq 0\right)$ denotes the bracket i.e. the quadratic variation process of $\left(\mathcal{E}_{s}, s \geq 0\right)$ and we have :

$$
d<\mathcal{E}>_{s}=\mathcal{E}_{s}^{2} d s
$$

The Itô-Tanaka formula yields, for $K \leq 1$ :

$$
E\left(\left(K-\mathcal{E}_{t}\right)^{+}\right)=\frac{1}{2} E\left(L_{t}^{K}\right)
$$

As a consequence of (2.21), we obtain point $i$ ) of the following :

\section{Proposition 2.7}

i) The probability $\gamma$ on $[0,1] \times\left[0, \infty\left[\right.\right.$ admits a density $f_{\gamma}$ which satisfies :

$$
\gamma(d K, d t)=\frac{1}{2}\left(\frac{\partial^{2}}{\partial K \partial t} E\left(L_{t}^{K}\right)\right) d K d t \quad(0 \leq K \leq 1, t \geq 0)
$$

ii) A closed form of $f_{\gamma}$ is :

$$
f_{\gamma}(K, t)=\frac{1}{2 \sqrt{2 \pi K t}}\left(\frac{1}{2}-\frac{\log K}{t}\right) \exp \left(-\frac{(\log K)^{2}}{2 t}-\frac{t}{8}\right) 1_{[0,1]}(K) \cdot 1_{[0, \infty[}(t)
$$

\section{Proof of point ii) of Proposition 2.7}

In [6], we obtained the following explicit formula for $E\left(L_{t}^{K}\right)$ :

$$
E\left(L_{t}^{K}\right)=\frac{\sqrt{K}}{\sqrt{2 \pi}} \int_{0}^{t} \frac{d s}{\sqrt{s}} \exp \left(-\frac{(\log (K))^{2}}{2 s}-\frac{s}{8}\right)
$$

Hence :

$$
\frac{\partial^{2}}{\partial K \partial t} E\left(L_{t}^{K}\right)=\frac{1}{\sqrt{2 \pi t}} \frac{\partial}{\partial K}\left(\sqrt{K} \exp \left(-\frac{(\log K)^{2}}{2 t}-\frac{t}{8}\right)\right)
$$

and (2.23) is an easy consequence of (2.25) and (2.22).

2.2.4 Relation with a result by N. Eisenbaum (see [2a] and [2b]).

We now relate the above description of $\gamma$ as in Proposition 2.7 with the definition-formula established in $[2 \mathrm{~b}]$ :

$$
\int_{-\infty}^{\infty} \int_{0}^{t} f(K, s) d_{K, s}\left(L_{s}^{K}\right)=\int_{0}^{t} f\left(\mathcal{E}_{s}, s\right) d \mathcal{E}_{s}+\int_{1-t}^{1} f\left(\mathcal{E}_{1-s}, 1-s\right) d_{s} \mathcal{E}_{1-s} \quad(t \leq 1)
$$

This formula which is the translation for $X_{s}=\mathcal{E}_{s}$ of the formula found in Theorem 2.2 of $[2 \mathrm{~b}]$ for a general reversible semi-martingale. Here, on the RHS of (2.26), the second stochastic integral is taken with respect to the natural filtration of $\widehat{\mathcal{E}}_{s}=\mathcal{E}_{1-s}$, which is, of 
course, that of $\widehat{B}_{s}=B_{1-s}$. We take $f$ bounded, Borel, with support in $[0,1]_{K} \times[0,1]_{s}$. In order to relate formula (2.26) with Proposition 2.7, we note that :

a) $\int_{0}^{1} \int_{0}^{1} f(K, s) \gamma(d K, d s)=\frac{1}{2} E\left(\int_{0}^{1} \int_{0}^{1} f(K, s) d_{K, s}\left(L_{s}^{K}\right)\right)$

which follows from (2.21), and the monotone class Theorem.

b) From formula (2.26) and the fact that $\left(\mathcal{E}_{s}, t \geq 0\right)$ is a martingale, we deduce :

$$
E\left(\int_{0}^{1} \int_{0}^{1} f(K, s) d_{K, s}\left(L_{s}^{K}\right)\right)=E\left(\int_{0}^{1} f\left(\widehat{\mathcal{E}}_{s}, 1-s\right) d \widehat{\mathcal{E}}_{s}\right)
$$

which we shall compute explicitely thanks to the semi martingale decomposition of $\left(\widehat{\mathcal{E}}_{s}, s \leq 1\right)$ in its own filtration. This is presented in the next :

\section{Proposition 2.8}

i) The canonical decomposition of $\left(B_{t}, t \leq 1\right)$ in the filtration $\mathcal{B}_{t}^{(1)}:=\sigma\left(B_{s}, s \leq t\right) \vee \sigma\left(B_{1}\right)$ is :

$$
B_{t}=B_{t}^{*}+\int_{0}^{t} \frac{B_{1}-B_{s}}{1-s} d s
$$

where $\left(B_{t}^{*}, t \leq 1\right)$ is a $\left(\mathcal{B}_{t}^{(1)}, t \leq 1\right)$ Brownian motion.

ii) The canonical decomposition of $\widehat{B}_{t}=B_{1-t}$ in its own filtration is :

$$
\widehat{B}_{t}=B_{1-t}=B_{1}+\beta_{t}^{*}-\int_{0}^{t} \frac{B_{1-s}}{1-s} d s
$$

where $\left(\beta_{t}^{*}, t \leq 1\right)$ is a Brownian motion in $\left\{\widehat{\mathcal{B}}_{t}:=\sigma\left(\widehat{B}_{u}, u \leq t\right), t \leq 1\right\}$.

iii) The canonical decomposition of $\widehat{\mathcal{E}}_{t}$ in $\widehat{\mathcal{B}}_{t}$ is :

$$
d \widehat{\mathcal{E}}_{t}=\widehat{\mathcal{E}}_{t}\left\{d \beta_{t}^{*}+d t\left(1-\frac{B_{1-t}}{1-t}\right)\right\}
$$

\section{Proof of Proposition 2.8}

i) is well-known, see, e.g., Jeulin-Yor ([4], 1979) or Itô (1976) : Extension of Stochastic Integrals $([3])$

ii) may be deduced from $i$ ), when $i$ ) is applied to $\beta_{t}=B_{1-t}-B_{1}$. Actually, formula (2.29) appears in [2a], bottom of p. 308

iii) follows from ii), thanks to Itô's formula. 
Comments a) We are grateful to N. Eisenbaum (personal communication) for pointing out formula (2.29), which allowed to correct our original wrong derivation of the canonical decomposition of $\widehat{B}_{t}=B_{1-t}$ :

$$
\widehat{B}_{t}=B_{1-t}=B_{1}+\widetilde{\beta}_{t}+\int_{0}^{t} \frac{d u}{u}\left(\widehat{B}_{u}-B_{1}\right)
$$

Indeed, by time-reversal from (2.28) in Proposition 2.8, there is the identity :

$$
\widehat{B}_{t}=B_{1-t}=B_{1}+\widetilde{\beta}_{t}+\int_{0}^{t} \frac{d u}{u}\left(\widehat{B}_{u}-B_{1}\right)
$$

where $\widetilde{\beta}_{t}=B_{1-t}^{*}-B_{1}^{*}$ is a Brownian motion, but is $\underline{\text { not }}$ the canonical decomposition of $\widehat{B}_{t}$ in $\widehat{\mathcal{B}}_{t}$ (for a discussion of non-canonical decomposition of Brownian motion, see, e.g. Yor [9], Chiu [10], Hibino, Hitsuda, Muraoka [11]).

b) A slightly different derivation of (2.29) consists in remarking that $B_{1-t}=(1-t) B_{1}+b(t)$ with $(b(t), 0 \leq t \leq 1)$ a standard Brownian bridge.

From $i$ ), this Brownian bridge admits the decomposition :

$$
b(t)=\beta_{t}^{*}-\int_{0}^{t} \frac{b(s)}{1-s} d s
$$

Thus we obtain :

$$
\begin{aligned}
B_{1-t} & =(1-t) B_{1}+\beta_{t}^{*}-\int_{0}^{t} \frac{d s}{1-s}\left(b(s)+(1-s) B_{1}-(1-s) B_{1}\right) \\
& =(1-t) B_{1}+\beta_{t}^{*}-\int_{0}^{t} \frac{B_{1-s}}{1-s} d s+t B_{1} \\
& =B_{1}+\beta_{t}^{*}-\int_{0}^{t} \frac{B_{1-s}}{1-s} d s
\end{aligned}
$$

which is precisely $(2.29)$.

Now, plugging (2.30) in (2.27), and with the help of $i$ ), we get :

$$
\begin{aligned}
\int_{0}^{1} \int_{0}^{1} f(K, s) \gamma(d K, d s) & =\frac{1}{2} E\left(\int_{0}^{1} f\left(\mathcal{E}_{s}, s\right) \mathcal{E}_{s}\left(1-\frac{B_{s}}{s}\right) d s\right) \\
& =\frac{1}{2} E\left(\int_{0}^{1} \mathcal{E}_{s} f\left(\mathcal{E}_{s}, s\right)\left(\frac{1}{2}-\frac{\log \mathcal{E}_{s}}{s}\right) d s\right)
\end{aligned}
$$

which matches perfectly with our previous formula (2.23). Thus, we have established a close link with local time-space calculus as developed in $([2 \mathrm{a}]-[2 \mathrm{~b}])$. 


\subsection{An extension of Theorem 2.1}

In the next statement, we shall replace the standard Brownian martingale $\left(\mathcal{E}_{t}, t \geq 0\right)$ by the semimartingale $\left(\mathcal{E}_{t}^{\sigma,-\nu}:=\exp \left(\sigma B_{t}-\nu t\right), t \geq 0\right) \quad(\sigma \neq 0, \nu>0)$. Then we can show :

Theorem 2.9 i) There exists a probability on $[0,1] \times\left[0, \infty\left[\right.\right.$, which we shall denote by $\gamma_{\sigma, \nu}$ such that:

$$
\Pi_{\sigma, \nu}(K, t):=E\left(\left(K-\mathcal{E}_{t}^{\sigma,-\nu}\right)^{+}\right)=\gamma_{\sigma, \nu}([0, K] \times[0, t])
$$

ii) Moreover, $\gamma_{\sigma, \nu}$ is the law of:

$$
\left(U, Y_{\frac{\nu}{|\sigma|}}, \frac{1}{|\sigma|} \log \frac{1}{U}\right)
$$

where $U$ is uniform on $[0,1]$, independent of $\left(Y_{a, b}, a, b>0\right)$ as introduced in Lemma 2.2.

\section{Proof of Theorem 2.9}

We may choose $\sigma>0$, since $\sigma B_{t} \stackrel{(\text { law })}{=}-\sigma B_{t}$. Then, from the definition (2.26), we write, for $K \leq 1$, by Fubini :

$$
\begin{aligned}
E\left(\left(K-\mathcal{E}_{t}^{\sigma,-\nu}\right)^{+}\right) & =\int_{0}^{K} P\left(\mathcal{E}_{t}^{\sigma,-\nu} \leq x\right) d x \\
& =\int_{0}^{K} P\left(B_{1}<\frac{\nu \sqrt{t}}{\sigma}+\frac{\log x}{\sigma \sqrt{t}}\right) d x \\
& =\int_{0}^{K} \mathcal{N}\left(\frac{\nu \sqrt{t}}{\sigma}+\frac{\log x}{\sigma \sqrt{t}}\right) d x \\
& =\int_{0}^{K} P\left(Y_{\frac{\nu}{\sigma}, \frac{1}{\sigma} \log \frac{1}{x}} \leq t\right) d x \quad \text { (from Lemma 2.2) }
\end{aligned}
$$

which implies points $i$ ) and $i$ ) of Theorem 2.9 .

Note that (2.33) corresponds to the first description in Section 2.2 of the particular case $\sigma=1, \nu=\frac{1}{2}$. We would like to see whether there is a second description of $\gamma_{\sigma, \nu}$; in particular, what is the law of $Y_{\frac{\nu}{\sigma}}, \frac{1}{\sigma} \cdot \log \frac{1}{U}$ ? Let us denote by $f_{\sigma, \nu}$ the density of $Y_{\frac{\nu}{\sigma}}, \frac{1}{\sigma} \cdot \log \frac{1}{U}$. Then, we have :

Proposition 2.10 The following formulae hold :

$$
\text { i) } f_{\sigma, \nu}(t)=\frac{\sigma}{2 \sqrt{2 \pi t}} e^{-\frac{\nu^{2} t}{2 \sigma^{2}}}\left(1+\left(2 \frac{\nu}{\sigma^{2}}-1\right) \int_{0}^{\infty} e^{-\mu x-\frac{x^{2}}{2 \sigma^{2} t}} d x\right)
$$

where $\mu=1-\frac{\nu}{\sigma^{2}}$

ii) In particular, if $\frac{2 \nu}{\sigma^{2}}=1$, (this condition ensures that $\left(\mathcal{E}_{t}^{\sigma,-\nu}, t \geq 0\right)$ is a martingale!), we have :

$$
f_{\sigma, \frac{\sigma^{2}}{2}}(t)=\frac{\sigma}{2 \sqrt{2 \pi t}} e^{-\frac{\sigma^{2} t}{8}}
$$


$\left(=f_{4 B_{1}^{2}}(t)\right.$ if $\sigma=1$ and $\nu=\frac{1}{2}$, in agreement with Proposition 2.5, formula (2.17))

\section{Proof of (2.34)}

From (2.12) and (2.33) we have :

$$
f_{\sigma, \nu}(t) d t=\frac{1}{2} \int_{0}^{1} d u\left\{P\left(T_{\frac{1}{\sigma} \log \frac{1}{u}}^{\left(\frac{\nu}{\sigma}\right)} \in d t\right)+P\left(G_{\frac{1}{\sigma} \log \frac{1}{u}}^{\left(\frac{\nu}{\sigma}\right)} \in d t\right)\right\}
$$

Making the change of variable $\log \frac{1}{u}=x$ and using (2.10) and (2.11), we obtain :

$$
\begin{aligned}
f_{\sigma, \nu}(t) & =\frac{1}{2} \int_{0}^{\infty} e^{-x}\left\{\left(\frac{x}{\sigma \sqrt{2 \pi t^{3}}}+\frac{\nu}{\sigma \sqrt{2 \pi t}}\right) e^{-\frac{(x-\nu t)^{2}}{2 \sigma^{2} t}}\right\} d x \\
& =\frac{\sigma}{2 \sqrt{2 \pi t}} \exp \left(-\frac{\nu^{2} t}{2 \sigma^{2}}\right) \int_{0}^{\infty} \exp \left(-x-\frac{x^{2}}{2 \sigma^{2} t}+\frac{x \nu}{\sigma^{2}}\right)\left(\frac{x}{t \sigma^{2}}+\frac{\nu}{\sigma^{2}}\right) d x
\end{aligned}
$$

We now introduce the parameter $\mu=1-\frac{\nu}{\sigma^{2}}$ and we compute the integral :

$$
\begin{aligned}
I & :=\int_{0}^{\infty} e^{-\mu x-\frac{x^{2}}{2 \sigma^{2} t}}\left(\frac{x}{t \sigma^{2}}+\frac{\nu}{\sigma^{2}}\right) d x \\
& :=I_{1}+\frac{\nu}{\sigma^{2}} I_{2} \quad \text { with } \\
I_{1} & =\int_{0}^{\infty} e^{-\mu x-\frac{x^{2}}{2 \sigma^{2} t}} \frac{x}{t \sigma^{2}} d x=1-\mu \int_{0}^{\infty} e^{-\mu x-\frac{x^{2}}{2 \sigma^{2} t}} d x
\end{aligned}
$$

(after integrating by parts). Finally :

$$
\begin{aligned}
I & =I_{1}+\frac{\nu}{\sigma^{2}} I_{2}=1-\mu I_{2}+\frac{\nu}{\sigma^{2}} I_{2} \\
& =1+\left(\frac{2 \nu}{\sigma^{2}}-1\right) \int_{0}^{\infty} e^{-\mu x-\frac{x^{2}}{2 \sigma^{2} t}} d x \text { and : } \\
f_{\sigma, \nu}(t) & =\frac{\sigma}{2 \sqrt{2} \pi t} \exp \left(-\frac{\nu^{2} t}{2 \sigma^{2}}\right)\left\{1+\left(\frac{2 \nu}{\sigma^{2}}-1\right) \int_{0}^{\infty} e^{-\mu x-\frac{x^{2}}{2 \sigma^{2} t}} d x\right\}
\end{aligned}
$$

This proves (2.33).

\section{$2.4 \gamma$ as a signed measure on $\mathbb{R}_{+} \times \mathbb{R}_{+}$}

In this paragraph, we extend the definition of $\gamma$ to $[0, \infty[\times[0, \infty[$.

\section{Proposition 2.11}

i) There exists a signed measure $\gamma$ on $[0, \infty[\times[0, \infty[$ such that :

$$
\Pi_{\mathcal{E}}(K, t):=E\left(\left(K-\mathcal{E}_{t}\right)^{+}\right)=\gamma([0, K] \times[0, t])
$$

holds for all values $K, t \geq 0$. 
ii) $\gamma$ admits on $\mathbb{R}_{+} \times \mathbb{R}_{+}$the density $f_{\gamma}$ given by :

$$
\begin{aligned}
f_{\gamma}(K, t) & =\frac{1}{2 \sqrt{2 \pi K t}}\left(\frac{1}{2}-\frac{\log K}{t}\right) \exp \left(-\frac{(\log K)^{2}}{2 t}-\frac{t}{8}\right) 1_{[0, \infty[\times[0, \infty[}(K, t) \\
& =\frac{1}{2 \sqrt{2 \pi t}}\left(\frac{1}{2}+\frac{\log \frac{1}{K}}{t}\right) \exp \left(-\frac{1}{2 t}\left(\log \frac{1}{K}-\frac{t}{2}\right)^{2}\right) 1_{[0, \infty[\times[0, \infty[}(K, t)
\end{aligned}
$$

iii) Consequently, if $\gamma=\gamma^{+}-\gamma^{-}$is the decomposition of $\gamma$ into its positive and negative parts, we have:

$$
\gamma^{+}(d K, d t)=1_{\left(K \leq e^{\frac{t}{2}}\right)} \gamma(d K, d t)
$$

In particular :

$$
\gamma_{\mid(K, t ; K \leq 1)}^{+}=\gamma_{\mid(K, t) ; K \leq 1)}
$$

is a probability.

iv) Formula (2.31) may be synthetized as follows :

- if $K<1$ :

$$
\gamma(d K, d t)=\frac{d K}{2}\left\{P\left(G_{\log \left(\frac{1}{K}\right)}^{\left(\frac{1}{2}\right)} \in d t\right)+P\left(T_{\log \left(\frac{1}{K}\right)}^{\left(\frac{1}{2}\right)} \in d t\right)\right\}
$$

- if $K>1$ :

$$
\gamma(d K, d t)=\frac{d K}{2}\left\{P\left(G_{\log \left(\frac{1}{K}\right)}^{\left(\frac{1}{2}\right)} \in d t\right)-P\left(T_{\log \left(\frac{1}{K}\right)}^{\left(\frac{1}{2}\right)} \in d t\right)\right\}
$$

\section{Proof of Proposition 2.11}

Points i) and ii) are easy to prove. Indeed, for every $K \geq 0$ :

$$
E\left(\left(K-\mathcal{E}_{t}\right)^{+}\right)=\int_{0}^{K} P\left(\mathcal{E}_{t}<x\right) d x=\int_{0}^{K} \mathcal{N}\left(\frac{\sqrt{t}}{2}+\frac{\log x}{\sqrt{t}}\right) d x
$$

This last formula implies the existence of $\gamma$ and the density $f_{\gamma}$ of $\gamma$ is given by :

$$
\begin{aligned}
f_{\gamma}(K, t) & =\frac{\partial^{2}}{\partial K \partial t} \int_{0}^{K} \mathcal{N}\left(\frac{\sqrt{t}}{2}+\frac{\log x}{\sqrt{t}}\right) d x \\
& =\frac{\partial}{\partial t} \mathcal{N}\left(\frac{\sqrt{t}}{2}+\frac{\log K}{\sqrt{t}}\right) \\
& =\frac{1}{2 \sqrt{2 \pi K t}}\left(\frac{1}{2}-\frac{\log K}{t}\right) \exp \left(-\frac{(\log K)^{2}}{2 t}-\frac{t}{8}\right)
\end{aligned}
$$


Point iii) follows immediately.

Formulae (2.39) and (2.40) are obtained as in (2.12). Moreover, this time, we also need to use formulae from Theorem 1 of [6].

- If $A>1$, then :

$$
P\left(T_{\log A}^{(\nu)} \leq t\right)=E\left(\mathcal{E}_{t}^{(2 \nu)} 1_{\mathcal{E}_{t}^{(2 \nu)}>A^{2 \nu}}\right)+A^{2 \nu} P\left(\mathcal{E}_{t}^{(2 \nu)}>A^{2 \nu}\right)
$$

- If $A<1$ :

$$
P\left(T_{\log A}^{(\nu)} \leq t\right)=E\left(\mathcal{E}_{t}^{(2 \nu)} 1_{\mathcal{E}_{t}^{(2 \nu)}<A^{2 \nu}}\right)+A^{2 \nu} P\left(\mathcal{E}_{t}^{(2 \nu)}<A^{2 \nu}\right)
$$

(see point iii) in Theorem 1 of [6]). 


\section{References}

[1] A. Bentata, M. Yor From Black-Scholes and Dupire formulae to last passage times of local martingales. Notes from a course at the Bachelier Seminar, (IHP, 02/08). Prepublication LPMA, Paris VI, n¹223, (June 2008).

[2a] N. Eisenbaum Integration with respect to local time. Potential Analysis, 13, p. 303-328, (2000).

[2b] N. Eisenbaum Local-Time Space Calculus for Reversible semimartingales. Seminaire de Probabilités XL, p. 137-146, Springer, (2007).

[3] K. Itô Extension of stochastic integrals. Proceeding of the international Symposium on Stochastic Differential Equations (Res. Inst. Math. Sci. Kyoto Univ.), Kyoto (1976), p. 95-109, Wiley New-York, (1978).

[4] T. Jeulin, M. Yor Inégalités de Hardy, semimartingales et faux amis. Séminaire de Prob., XIII, LNM nº 721, p. 332-359, (1979).

[5] D. Madan, B. Roynette, M. Yor, (2008, a) Option Prices as Probabilities. Finance Research Letters, 5, p. 79-87.

[6] D. Madan, B. Roynette, M. Yor, (2008, b) An alternative expression for the BlackScholes formula in terms of first and last passage times. Preprint 2008, $\mathrm{n}^{\circ}$ 8, IEC Nancy.

[7] D. Madan, B. Roynette, M. Yor, (2008, c) From Black-Scholes formula to local times and last passages times for certain submartingales. Preprint 2008, $\mathrm{n}^{\circ}$ 14, IEC Nancy.

[8] D. Madan, B. Roynette, M. Yor, (2008, d) Unifying Black-Scholes type formulae which involve last passage times up to a finite horizon. Asia-Pacific Financial Markets, To appear.

[9] M. Yor Some aspects of Brownian motion, Part I, Some special functionals. Lectures in Math. ETH Zürich, Birkhäuser Verlag Basel, (1992).

[10] Y. Chiu From an example of Lévy. Séminaire de Prob., XXIX, LNM, n 1613, p. 162165, Springer , (1995).

[11] Y. Hibino, M. Hitsuda, H. Muraoka Construction of non-canonical representations of a Brownian motion. Hiroshima Math. J., $\mathrm{n}^{\circ}$ 3, p. 439-448, (1997). 